\title{
Phenotypic changes of lymphocyte populations in psoriasiform dermatitis animal model
}

\author{
MIHAELA SURCEL ${ }^{1,2}$, RADU-IONUȚ HUICA ${ }^{1,3}$, ADRIANA NARCISA MUNTEANU ${ }^{1}$, GHEORGHIȚA ISVORANU ${ }^{1}$, \\ IOANA RUXANDRA PÎRVU ${ }^{1}$, DAN CIOTARU ${ }^{1}$, CAROLINA CONSTANTIN $^{1,4}$, OVIDIU BRATU $^{3}$, \\ CONSTANTIN CĂRUNTU ${ }^{3}$, MONICA NEAGU ${ }^{1,2,4}$ and CORNEL URSACIUC ${ }^{1}$ \\ ${ }^{1}$ Immunobiology Laboratory, 'Victor Babeș' National Institute of Pathology, 050096 Bucharest; ${ }^{2}$ Faculty of Biology, \\ University of Bucharest, 050095 Bucharest; ${ }^{3}$ Department of Urology, 'Carol Davila' University of Medicine and \\ Pharmacy, 020021 Bucharest; ${ }^{4}$ Department of Pathology, Colentina University Hospital, 020125 Bucharest, Romania
}

Received July 6, 2018; Accepted August 24, 2018

DOI: $10.3892 / \mathrm{etm} .2018 .6978$

\begin{abstract}
Psoriasis is a T cell mediated, chronic inflammatory autoimmune skin disease that affects up to $2-3 \%$ of the global population and leads to a decrease in quality of life. Experimental data accumulated in recent years highlighted the important role played by the immune system in the pathogenesis of this disease. Non-human psoriasis models are an important research tool that attempts to reproduce the clinical features of the disease in order to explain the pathogenesis of psoriasis and to identify possible therapeutic targets. Imiquimod-based murine model of psoriatic dermatitis is an alternative to traditional models of experimental psoriasis in mice and the induced dermatitis closely mimics the pathologic changes in human psoriasis. In order to emphasize changes in immune cell populations involved in lesion pathogenesis, we performed a murine model of psoriasiform dermatitis model by topical IMQ application. The progress and the
\end{abstract}

Correspondence to: Dr Monica Neagu, Immunobiology Laboratory, 'Victor Babes' National Institute of Pathology, 99-101 Splaiul Independentei, 050096 Bucharest, Romania

E-mail: neagu.monica@gmail.com

Abbreviations: ADAMTSL5, A disintegrin and metalloproteinase with thrombospondin-like protein 5; BD, Becton-Dickinson; CD, cluster of differentiation; DLQI, Dermatology Life Quality Index; FACS, fluorescence-activated cell sorting; FBS, fetal bovine serum; $\mathrm{H} \& \mathrm{E}$, haematoxylin and eosin; IFN, interferon; IL, interleukin; IMQ, imiquimod; K2-EDTA, kalium 2 ethylenediamine tetra-acetate; LFA-1, lymphocyte function-associated antigen-1; NF- $\kappa \mathrm{B}$, nuclear factor $\kappa$-light-chain-enhancer of activated $\mathrm{B}$ cells; $\mathrm{NK}$, natural killer; PASI, Psoriasis Area Severity Index; PBMC, peripheral blood mononuclear cells; PE, phycoerythrin; $\mathrm{PE} / \mathrm{Cy}$, phycoerythrin complex with cyanine; PerCP/Cy, peridinin chlorophyll protein complex with cyanine; RPMI, Roswell Park Memorial Institute; SD, standard deviation; Th, Helper T cells; TLR, Toll-like receptor; TNF, tumor necrosis factor

Key words: psoriasiform dermatitis, imiquimod, murine model, inflammation, lymphocytes severity of IMQ-induced skin inflammation were clinically (PASI score) and histopathologically evaluated. The immunological changes induced by IMQ treatment in lymphocyte populations from peripheral blood and spleen were evaluated by flow cytometry. The main changes observed in peripheral blood were the significantly increased T-CD8 $\mathrm{a}^{+}$lymphocyte and $\mathrm{NK} 1.1^{+}$cell percentages and the decreased $\mathrm{T}-\mathrm{CD} 4^{+}$and B lymphocyte percentages in IMQ-treated mice. In spleen samples, lymphocytes showed the same tendency of variation as in peripheral blood, but without statistical significance. A significant decrease of B cells percentages was observed in spleen suspensions. Data obtained in skin samples may suggest the involvement of $\mathrm{CD} 3 \varepsilon^{+}, \mathrm{CD}^{+}$and $\mathrm{CD} 8 \mathrm{a}^{+}$cells in the lesional process. This murine model was analyzed by performing a basic cellular profile at three levels: peripheral blood, spleen and skin. The evaluation aimed to establish the immune framework of this experimental model that could further be used for etipathogenic mechanism identification and/or for studies regarding targeted therapies.

\section{Introduction}

Psoriasis is a T cell mediated, chronic inflammatory autoimmune skin disease (1) with joint manifestations, that affects up to $2-3 \%$ of the global population (2) and leads to a decrease in quality of life (3). Psoriasis is associated with psoriatic arthritis (4) and depressive illness $(5,6)$. Several cardiometabolic pathologies (7) are also associated with psoriasis, including obesity (8), dislipidemia (9), diabetes (10) and venous thromboembolism (11).

The causes of psoriasis are not fully understood; genetic factors and an inneffective immune system seem to be the main factors involved in the etiology of this pathology. Other aggravating factors are skin trauma, streptococcal infections, endocrine factors, certain medications, emotional stress, smoking, alcohol and environmental factors (12).

From a clinical point of view, psoriasis can be divided in two main groups: non-pustular (psoriasis vulgaris, guttate, erythrodermic, palmoplantar, psoriatic arthritis and inverse psoriasis) and pustular psoriasis (generalized pustular psoriasis, impetigo herpetiformis and localized pustular psoriasis) (13). 
These clinical variants have common features consisting of erythema, skin scaling and thickening. The assessment of psoriasis severity and evolution is based on clinical indicators (PASI score) (14), but also on the quality of life of the patient (DLQI score) (15). The clinical type of psoriasis and the severity of the psoriatic lesions are important elements in establishing the treatment in this disease. There are three main types of therapies: Topical treatment (for mild forms), light therapy (for moderate to severe forms) and systemic medication (for severe forms) (16).

Clinical and experimental data accumulated in recent years highlighted the important role played by the immune system in the pathogenesis of psoriasis. Although it is considered a T-cell-mediated inflammatory disease, the pathogenesis of psoriasis involves cells belonging to both adaptive and innate immunity (dendritic cells, NK cells, macrophages) as well as non-immune cells (keratinocytes, endothelial cells) (17). The autoantigens that activate autoimmune reactions in psoriasis are not fully described. LL-37, an antimicrobial peptide produced by keratinocytes was found overexpressed in moderate to severe psoriatic forms (18). Recent studies reveal another possible autoantigen, ADAMTS-like protein 5, that is produced by melanocytes and was shown to activate the Th17 response (19).

Two phases are described in the pathogenesis of psoriasis: the initiation and the maintenance of the disease. Some triggering factors trigger the secretion of TNF- $\alpha$, IFN- $\gamma$ and IL- 6 by plasmacytoid dendritic cells, NK cells, macrophages and keratinocytes. These mediators activate myeloid dendritic cells that release IL-12 and IL-23. IL-12 leads to differentiation of naive $\mathrm{T}$ lymphocytes in Th1 lymphocytes, which will secrete IFN- $\gamma$ and TNF- $\alpha$; IL-23 causes differentiation of naive $\mathrm{T}$ lymphocytes in Th17 lymphocytes, which secrete IL-17A, IL-17F and IL-22. These mediators further activate keratinocytes that induce the production of antimicrobial peptides (LL-37), proinflammatory cytokines (TNF- $\alpha$, IL-1 $\beta$, IL-6), chemokines and S100 proteins, hence maintaining and propagating inflammation (20).

Due to the importance of immunological mechanisms in the pathogenesis of psoriasis, therapeutical research has been directed towards isolating compounds that block or inhibit these mechanisms. Clinical trials using biological agents that target specific components of the immune system are currently underway. There is a panel of drugs already approved or that are in the testing stage (21-26). Currently available biological therapies are: anti-TNF- $\alpha$ agents (Infliximab, Adalimumab, Etanercept), anti-LFA-1 (Efalizumab), IL-17 inhibitors (Brodalumab, Ixekizumab and Secukinumab), IL-23 inhibitors (Guselkumab), and IL-12/23 inhibitors (Ustekinumab) $(27,28)$.

Non-human psoriasis models are an important research tool that attempts to reproduce in vitro and in vivo the clinical features of the disease in order to explain the pathogenesis of psoriasis and to identify possible therapeutic targets. Animal models of psoriasis must reflect specific clinical features of the disease, including histological alterations, a similar pathogeny and a comparable response to therapeutic agents. Although murine models successfully mimic psoriasiform dermatitis, the differences between the two species (mouse vs. human) are a disadvantage of in vivo models. Traditional models of experimental psoriasis in mice, based on spontaneous mutations or genetic engineering, are defined by high costs and expertise.
A more accessible and easier-to-implement alternative is the induction of psoriasis-like skin lesions by the topical application of Imiquimod (IMQ), a nucleoside (1-isobutyl-1H-imidazo[4,5-c]quinolin-4-amine) analogue of imidazoquinoline family (29). It is an anti-viral and anti-neoplastic drug used in the treatment of genital warts, basal cell carcinoma and actinic keratosis. IMQ is a ligand of TLR7 and TLR8 (30), an activator of the NF- $\mathrm{KB}$ and, consequently, a potent immune modulator. IMQ mimics partially the pathogenic processes of psoriasis, acting at the interface between innate and acquired immunity both directly and indirectly (31). Direct action consists of binding of TLR7 and TLR8 on macrophages, monocytes and dendritic cells, and indirect action refers to the induction of pro-inflammatory cytokine secretion, such as IFN- $\gamma$, IL-6 and TNF- $\alpha$. IMQ increases the ability of Langerhans cells to present the antigen and stimulates their migration to the lymph nodes where the antigens are presented to T lymphocytes, thus activating the adaptive immune response. Topical application of IMQ induces increased IL- 6 and IL- 8 production by activated keratinocytes (32). IMQ-induced cytokine production elicits a Th1-weighted cellular immune response, enhancing the activation of cytotoxic T cells and inhibiting the Th2-mediated response (33). The IMQ-based model of psoriasiform dermatitis offers a series of advantages: it is relatively easy to use, control and reproduce, while the induced dermatitis closely mimics the pathologic changes in human psoriasis.

In order to emphasize changes in immune cell populations involved in lesion pathogenesis, we performed a murine model of psoriasiform dermatitis model by topical IMQ application. IMQ-induced skin inflammation and lesions were assessed using in vivo measurements and histological data, while the cellular evaluation for T, B and NK cell populations was performed by flow cytometry. The changes in these cell populations were analysed in peripheral blood, spleen and skin to observe the immune area affected by the pathological process. The evaluation was intended to establish the immune framework of this experimental model that could be further used for etipathogenic mechanisms identification and/or for studies regarding targeted therapies.

\section{Materials and methods}

Animal strain. C57BL/6 mice, purchased from Jackson Laboratory (Bar Harbor, ME, USA), were provided by the Animal Husbandry from Victor Babeş National Institute. The animals were maintained in a temperature-controlled, air-conditioned animal house $\left(20 \pm 4{ }^{\circ} \mathrm{C}, 55 \pm 10 \%\right.$ humidity) with a 12/12-light/dark cycle. The mice were housed in individual cages in an open cage system and received food and water ad libitum. The animals were monitored daily. The study protocol was approved by the Ethics Committee from 'Victor Babeș' Institute (Bucharest, Romania), and mouse handling was done in accordance with recognized principles of laboratory animal care in the framework of EU Directive 2010/63/EU for animal experiments (34).

IMQ-based murine model of psoriatic dermatitis. In order to induce psoriatic dermatitis, an IMQ-based murine model of psoriasiform dermatitis was replicated according to 
the protocols described in literature (35). Three groups of C57BL/6 mice were considered (1:1 sex ratio), 8-11 weeks old, with a mean weight of $21 \pm 3 \mathrm{~g}$ (Balance Scientech SL-3100D; Boulder, CO, USA), free of any medication in the previous $72 \mathrm{~h}$ : (i) the psoriasis group (10 mice) received a daily topical dose of $62.5 \mathrm{mg}$ IMQ cream (5\% Aldara Cream; Meda AB, Sweden) on the shaved back for 5 consecutive days (a daily dose of $3.125 \mathrm{mg}$ of active compound); (ii) the control group (10 mice) was treated similarly with vaseline-based cream (Locobase Cream, Astellas Pharma European BV, Leiderdorp, The Netherlands); and (iii) the normal group (15 mice) with no topical treatment.

Evaluation of IMQ-induced skin inflammation in vivo measurements. The progress and the severity of the inflammation of the back skin were monitored daily for erythema (redness), desquamation (skin scaling) and induration (thickening), on a scale from 0 to 4: 0 , none; 1 , slight; 2 , moderate; 3 , marked; and 4, very marked. A modified PASI score was calculated as a cumulative score (erythema + scaling + thickening; the affected area was not taken into account), on a range from 0 to 12 for each day of treatment.

Blood and tissues sampling. After 6 days from the experiment initiation all the animals were weighed and anesthetized with ketamine/acepromazine, $100 \mathrm{mg} / \mathrm{kg} / 5 \mathrm{mg} / \mathrm{kg}$ (Ketathesia; Henry Schein Animal Health, Dublin, OH, USA/Phoenix Pharmaceuticals, St. Joseph, MO, USA) prior to blood collection. Blood was collected by retro-orbital puncture in K2-EDTA coated tubes (Microvette; Sarstedt AG \& Co., Numbrecht, Germany). All the animals were sacrificed for spleens and skin sampling.

Assessing the splenomegaly. The spleens were weighed (Balance AEP-1500 A; Adam Equipment Co., Ltd., Milton Keynes, UK) separately in order to assess the splenomegaly by calculating the ratio of the weight of the spleen (SW) and bodyweight $(\mathrm{BW})$.

Histopathological evaluation. Skin samples removed from the back skin were fixed in $10 \%$ buffered formalin, embedded in paraffin, sectioned in $5 \mu \mathrm{m}$ thick sections, stained with haematoxylin and eosin $(\mathrm{H} \& \mathrm{E})$ and examined by pathologists.

Isolation of spleen cells. Single cell suspensions (for all groups of mice) were prepared from spleen by mechanical disruption; the spleens were harvested in RPMI-1640 media with 5\% FBS (Biochrom GmbH, Berlin, Germany) and passed through a $70 \mu \mathrm{m}$ cell strainer (BD Falcon; BD Biosciences, San Jose, CA, USA). The suspensions were centrifuged for $5 \mathrm{~min}$ at $350 \mathrm{~g}$ and resuspended in Cell Staining Buffer (BioLegend, San Diego, CA, USA) at $10^{6}$ cells $/ \mathrm{ml}$.

Preparation of skin cell suspensions. Skin cell suspensions (for test and control groups) were prepared from skin by mechanical disruption (Tissue Ruptor, Qiagen Istruments Hambrechtikon, Switzerland); the skin samples were harvested in RPMI-1640 media with 5\% FBS (Biochrom $\mathrm{GmbH}$ ) and passed through a $70 \mu \mathrm{m}$ cell strainer (BD Falcon; BD Biosciences) after the mechanical disruption. The suspensions were centrifuged for $5 \mathrm{~min}$ at $350 \mathrm{~g}$ and resuspended in Cell Staining Buffer (BioLegend) at $10^{6}$ cells $/ \mathrm{ml}$.

Flow cytometry analysis. Blood samples, spleen and skin cell suspensions (for considered groups of mice) were incubated with TruStain fcX (anti-mouse CD16/32, isotype Rat IgG2a, $\lambda$ ) antibody (BioLegend) in order to block non-specific antibody binding, then stained for $20 \mathrm{~min}$ at room temperature with the following antibodies: Alexa Fluor 647 anti-mouse CD3e (clone 145-2C11, isotype Armenian Hamster IgG) (BioLegend), Brilliant Violet 421 anti-mouse CD4 (clone GK1.5, isotype Rat IgG2b, $)$ (BD Horizon; BD Biosciences), Alexa Fluor 488 anti-mouse CD8a (clone 53-6.7, isotype Rat IgG2a, $\kappa$ ), PerCP/Cy5.5 anti-mouse CD19 (clone 6D5, isotype Rat IgG2a, $)$ (both from BioLegend) and Brilliant Violet 510 anti-mouse NK1.1 (clone PK136, isotype mouse IgG2a, $\kappa)$ (BD Horizon; BD Biosciences). Surface staining was followed by red blood cell lysis with BD Pharm Lyse (BD Biosciences) for $3 \mathrm{~min}$ at $37^{\circ} \mathrm{C}$ and centrifugation for $5 \mathrm{~min}$ at $350 \mathrm{~g}$. Cells were washed twice with cell staining buffer and analysed by flow cytometry. Unlabeled cells were used as controls; nonspecific fluorescence signals due to spectral overlap were automatically compensated.

Data acquisition was performed on MoFlo Astrios EQ cell sorter with Summit software (Beckman Coulter, Brea, CA, USA). Data analysis (blood and spleen suspensions samples) was performed using FlowJo v.10 software (TreeStar; BD Biosciences), with CD $3 \varepsilon^{+}$lymphocytes gated as $\mathrm{CD} 4{ }^{+} \mathrm{CD} 8 \mathrm{a}^{-}$and $\mathrm{CD} 4{ }^{-} \mathrm{CD} 8 \mathrm{a}^{+}$populations, and $\mathrm{CD} 3 \varepsilon^{-}$lymphocytes as $\mathrm{CD} 19^{+} \mathrm{NK} 1.1^{-}$and $\mathrm{CD} 19^{-} \mathrm{NK} 1.1^{+}$populations. The expression of CD3e, CD4, CD8a, CD19 and NK1.1 from skin samples was evaluated using Overton Subtraction performed with FlowJo v.10 software (TreeStar; BD Biosciences).

Statistical analysis. Data were analysed using Microsoft Excel (Microsoft, Redmond, CA, USA). The results were expressed as mean \pm SD. For skin samples, the expression of fluorescence levels was evaluated using Overton Subtraction technique performed with FlowJo v.10 software (TreeStar; BD Biosciences). The results were expressed as mean $\pm \mathrm{SD}$ calculated using the Overton Subtraction method. For statistical analysis, Student's t-test (two-tailed, assuming equal variance) was used to assess the differences between the experimental groups. P-values $<0.05$ were considered to indicate a statistically significant difference.

\section{Results}

Psoriasiform dermatitis experimental model. In order to assess whether topical IMQ application leads to psoriasis-specific skin lesions, we applied a daily topical dose of Aldara cream on the shaved back skin of 10 C57BL/6 mice for 5 consecutive days. For control, a group of $10 \mathrm{C} 57 \mathrm{BL} / 6$ mice were treated similarly with a vaseline-based cream.

The progress and the severity of the inflammation of the back skin were monitored daily for both groups of mice by scoring of erythema, desquamation and induration parameters, on a scale from 0 to 4 : 0 , None; 1 , slight; 2 , moderate; 3 , marked; 4 , very marked. Erythema was first observed in IMQ-treated mice on day 2; skin scaling and thickening became visible 

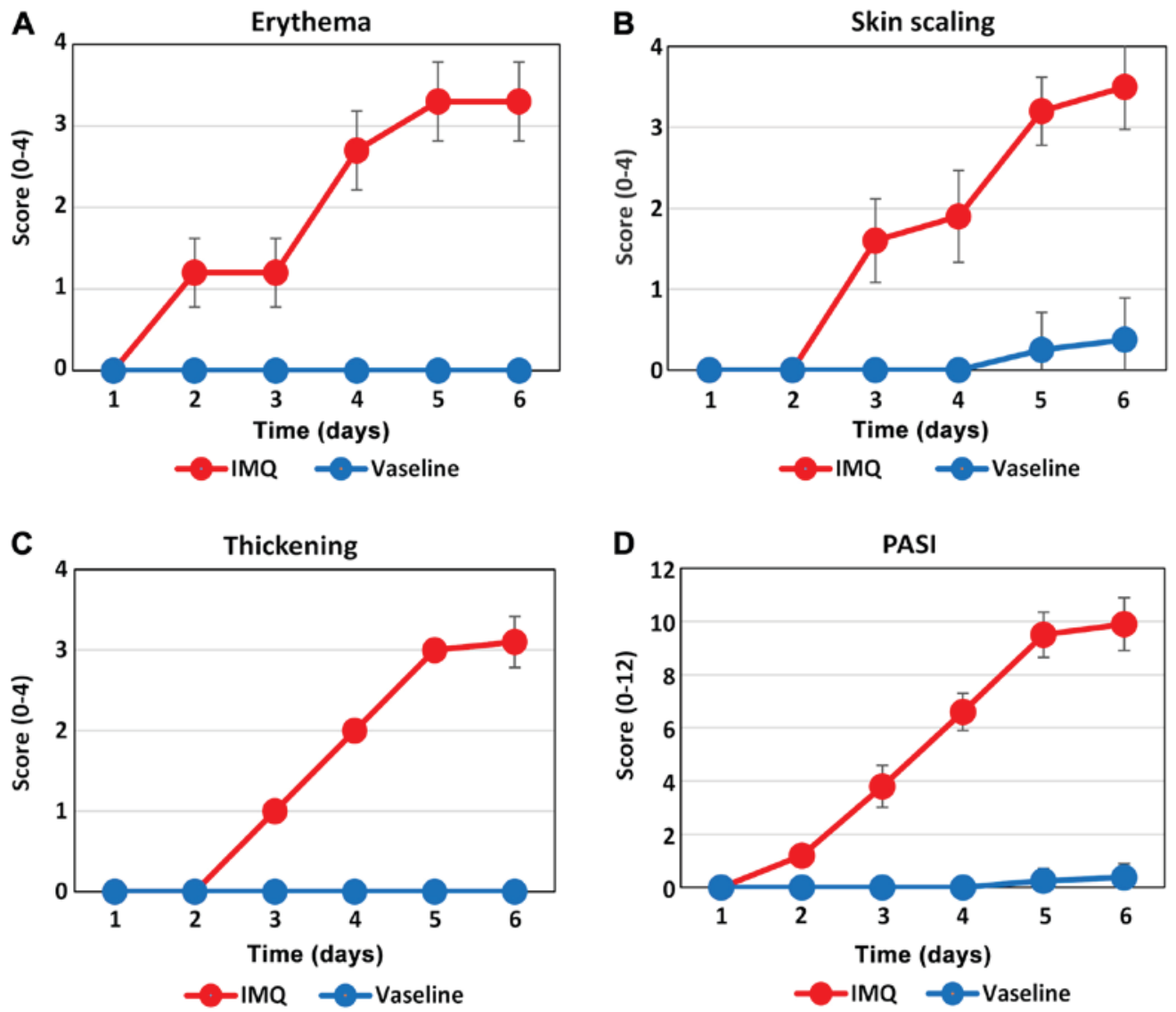

Figure 1. In vivo measurement-scoring for erythema, skin scaling and thickening; PASI cumulative score. C57BL/6 mice (test group) were treated daily with a topical dose of Aldara cream on the shaved back skin for 5 consecutive days. A control group was treated similarly with a vaseline-based cream. Erythema (A), skin scaling (B) and thickening (C) were scored daily (scale 0-4) and the cumulative PASI score (erythema plus skin scaling plus thickening) (D) was calculated daily for each group (test and control). The results are presented as mean \pm SD ( $\mathrm{n}=10$ for each test and control group).

A

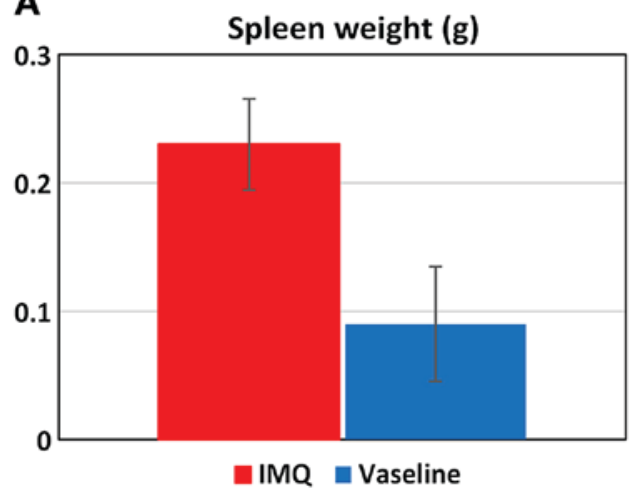

B

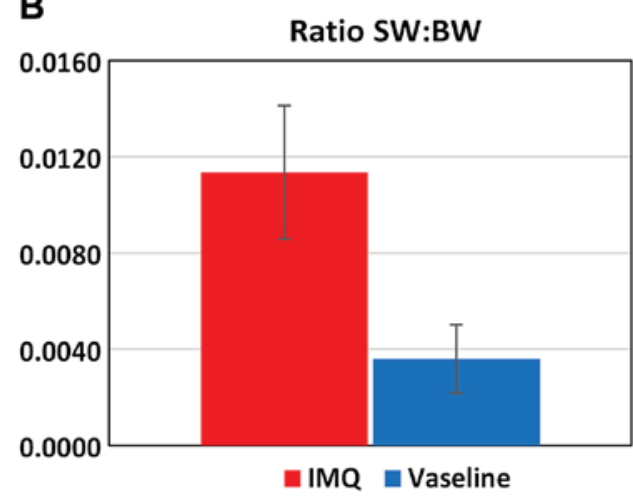

Figure 2. Splenomegaly assessment. C57BL/6 mice (test and control group) were weighed and sacrificed for spleens sampling. The spleens were weighed separately (A) and the ratio of the weight of the spleen (SW) and bodyweight (BW) was calculated (B). The results are presented as mean spleen weight \pm SD, respectively mean ratio $\mathrm{SW}: \mathrm{BW} \pm \mathrm{SD}$ ( $\mathrm{n}=10$ for each test and control group).

from day 3. Starting from day three, the inflammation of the skin became visible and continued to increase until the end of experiment. Mice treated with vaseline-based cream did not show any sign of inflammation. The independent scores for each parameter (erythema, skin scaling and thickening) and for each experimental group are presented in Fig. 1.

A modified PASI score was calculated as a cumulative score, including erythema, scaling and thickening scores, for each mouse from both groups, on a range from 0 to 12 for each day of experiment (Fig. 1A-C). The PASI score had a progressive evolution for IMQ-treated mice, reaching at the end of the experiment a value close to maximum (Fig. 1D).

At day 6 the experimental spleens from all the animals were weighed and splenomegaly was assessed by calculating the SW:BW ratio. For IMQ-treated mice the spleen weight was significantly higher compared to the vaseline-treated mouse group $\left(0.23 \pm 0.04\right.$ vs. $\left.0.09 \pm 0.04 ; \mathrm{P}<10^{-5}\right)($ Fig. $2 \mathrm{~A})$. The measurements showed that for IMQ-treated mice the spleen weight 

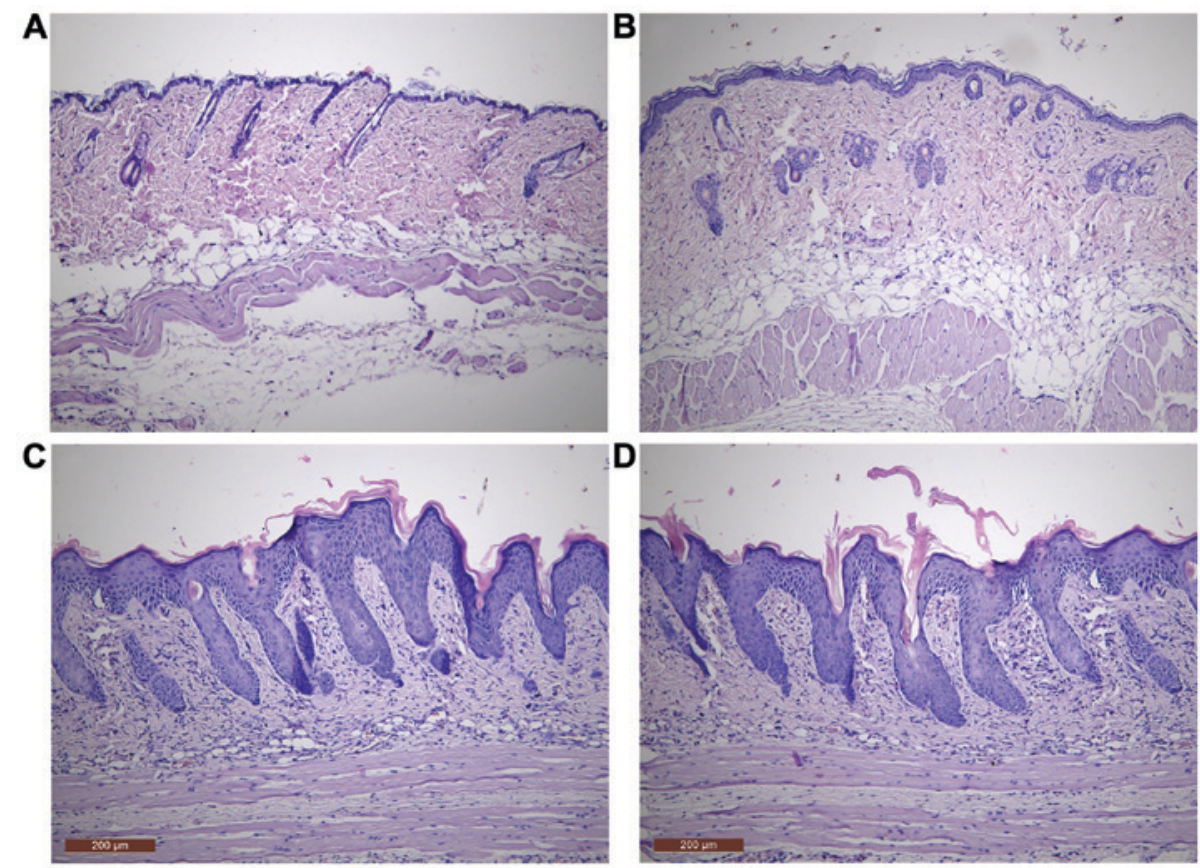

Figure 3. H\&E staining of skin samples harvested from dorsal area. (A) Normal mouse; (B) Vaseline-based cream treated mouse; (C and D) IMQ-treated mouse. (C and D) Histopathologic evaluation of the skin samples from IMQ-treated mice revealed hyperkeratosis, parakeratosis, acanthosis and elongation of rete ridges. (A and B) None of these histopathological findings were observed in control and normal group skin samples.

and the ratio $\mathrm{SW} / \mathrm{BW}$ is 2.5 times, respectively 3 times greater than in vaseline-treated mice $(0.0114 \pm 0.003$ vs. $0.0036 \pm 0.001$; $\mathrm{P}<10^{-5}$ ) (Fig. 2B).

Skin inflammation and the disease severity were histopathologically assessed. After 6 days, skin samples from each experimental group (test, normal and control) were harvested for histopathologic evaluation. IMQ treatment induced hyperkeratosis, parakeratosis, acanthosis and elongation of rete ridges (histopathological features that are typical assessed in human psoriatic skin samples) (Fig. 3C and D). None of these histopathological findings were observed in control (vaseline) and normal group (Fig. 3A and B).

Lymphocyte distribution inperipheralblood and spleen samples. To evaluate the immune cell pattern of the tested experimental model blood and spleen suspensions were assessed from all the groups analysed for T, B and NK cells population components by flow cytometry. Data analysis was performed using FlowJo v.10 software (TreeStar; BD Biosciences), with CD $3 \varepsilon^{+}$lymphocytes gated as $\mathrm{CD}^{+}{ }^{+} \mathrm{CD} 8 \mathrm{a}^{-}$and $\mathrm{CD} 4{ }^{-} \mathrm{CD} 8 \mathrm{a}^{+}$populations, and $\mathrm{CD} 3 \varepsilon^{-}$ lymphocytes as $\mathrm{CD} 19^{+} \mathrm{NK} 1.1^{-}$and $\mathrm{CD} 19^{-} \mathrm{NK} 1.1^{+}$populations. Unlabeled cells were used as controls; non-specific fluorescence signals due to spectral overlap were automatically compensated. Data were analysed using Microsoft Excel (Microsoft). The results were expressed as a percentage from the $\mathrm{CD}^{+}$lymphocytes (mean \pm SD) (Fig. 4).

In comparison to vaseline-treated mice and the normal group we noted in the IMQ-treated mice peripheral blood percentages of $\mathrm{T}_{-} \mathrm{CD} 4^{+}$and $\mathrm{B}$ lymphocytes decreased significantly while the percentages of $\mathrm{T}-\mathrm{CD} 8 \mathrm{a}^{+}$lymphocytes and NK $1.1^{+}$cells increased (Fig. 4A and B). The values obtained for the control groups (mice treated with vaseline-based cream and normal mice) are similar and the differences are not statistically significant. T-CD4 ${ }^{+} / \mathrm{T}-\mathrm{CD} 8 \mathrm{a}^{+}$ratio is lower in mice treated with IMQ compared to control groups and the differences between experimental groups are highly statistically significant $(\mathrm{P}<0.0005)$ (Fig. 4C).

To further investigate the immune cell pattern in the main lymphoid organ, we investigated immune cell populations in the spleen suspensions. The main change observed in spleen suspensions was the significant decrease of $B$ cells percentages in IMQ-treated mice $\left(35 \pm 11 ; \mathrm{P}<10^{-6}\right)$ compared to vaseline-treated mice (75 \pm 12$)$ and normal group (81 \pm 5$)$ (Fig. 5B). Other lymphocyte populations and subpopulations showed the same tendency of variation as in peripheral blood, but without statistical significance (Fig. 5A and B). T-CD4 ${ }^{+} / \mathrm{T}-$ $\mathrm{CD} \mathrm{a}^{+}$ratio is lower in mice treated with IMQ compared to control (vaseline) groups, but the differences are not statistically significant (Fig. 5C). The values obtained for the control (vaseline) groups (mice treated with vaseline-based cream and normal mice) were similar.

Analysis of fluorescence levels in skin samples. As skin is the main target tissue of the psoriatic mouse model, the effect of the IMQ treatment on $\mathrm{CD} 3 \varepsilon^{+}, \mathrm{CD} 4^{+}, \mathrm{CD} 8 \mathrm{a}^{+}, \mathrm{CD} 19^{+}$and $\mathrm{NK} 1.1^{+}$ cell populations in skin was assessed by flow cytometry. Analysis of lymphocyte populations in skin samples revealed a continuous fluorescence level which made impossible to gate positive and negative events. To highlight the differences between the fluorescence levels (stained vs. unstained samples) Overton subtraction was chosen and an increased value for Overton subtraction indicated a high level of fluorescence (Fig. 6).

Raised values of Overton subtraction were obtained for $\mathrm{CD} 3 \varepsilon^{+}$and $\mathrm{CD}^{+}$cells from skin samples of both IMQ and vaseline-treated mice, indicating a high level of fluorescence for these labelled cells. The differences between Overton subtraction values for experimental groups (IMQ-treated 
A

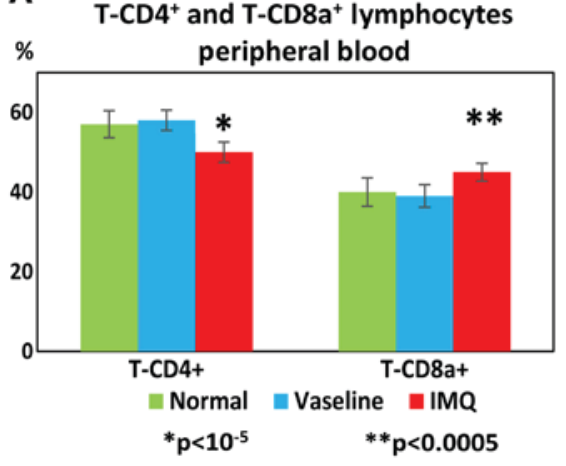

B

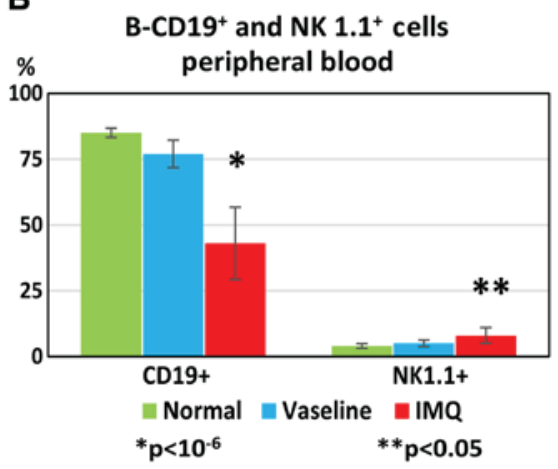

C

T-CD4+ / T-CD8a+ ratio peripheral blood

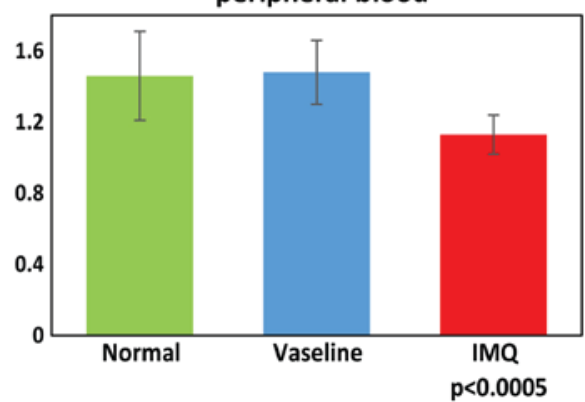

Figure 4. Distribution of lymphocyte populations in peripheral blood. (A) Percentage distribution of T-CD4 ${ }^{+}$and T-CD8a $\mathrm{a}^{+}$lymphocytes in IMQ-treated mice $\left(50 \pm 3,{ }^{*} \mathrm{P}<10^{-5}\right.$ and $\left.45 \pm 2,{ }^{* *} \mathrm{P}<0.0005\right)$ as compared to control (vaseline) (58 \pm 3 and $39 \pm 3$ ) and normal group (57 \pm 3 and $40 \pm 4$ ). (B) Percentage distribution of $\mathrm{CD} 19^{+}$lymphocytes and NK1.1 cells in IMQ-treated mice $\left(43 \pm 14,{ }^{*} \mathrm{P}<10^{-6}\right.$ and $\left.8 \pm 3,{ }^{* * *} \mathrm{P}<0.05\right)$ as compared to control (vaseline) (77 \pm 5 and $\left.5 \pm 1\right)$ and normal group (85 \pm 2 and $4 \pm 1)$. (C) Distribution of T-CD4 $+/ T-C D 8^{+}$ratio in IMQ-treated mice $(1.13 \pm 0.11, \mathrm{P}<0.0005)$ as compared to control (vaseline) $(1.48 \pm 0.18)$ and normal group $(1.46 \pm 0.25)$. The results are presented as a percentage from $\mathrm{CD} 3 \varepsilon^{+}$lymphocytes (mean $\pm \mathrm{SD}$ ).

A

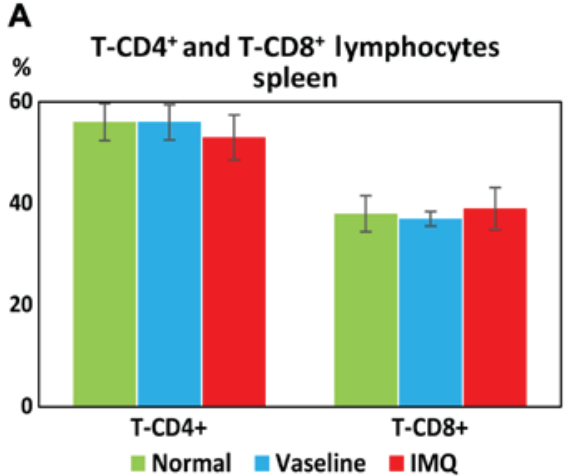

B

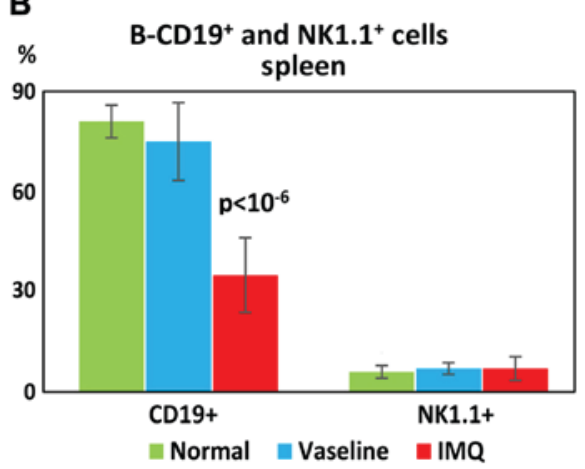

C

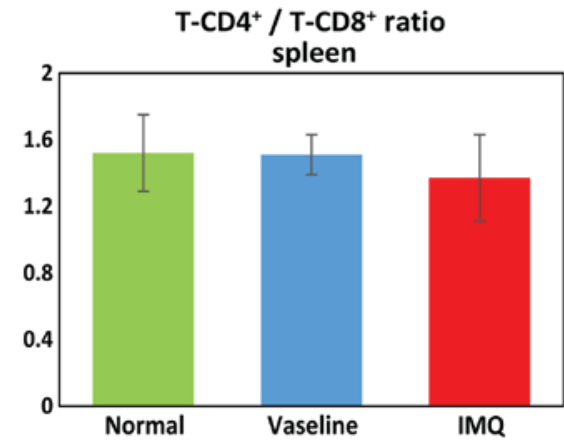

Figure 5. Distribution of lymphocyte populations in spleen. (A) Percentage distribution of T-CD4 ${ }^{+}$and T-CD $8 \mathrm{a}^{+}$lymphocytes in IMQ-treated mice (53 \pm 4 and $39 \pm 4$ ) as compared to control (vaseline) $(56 \pm 3$ and $37 \pm 1)$ and normal group (56 \pm 4 and 38 \pm 4 ). (B) Percentage distribution of CD19 $9^{+}$lymphocytes and NK1.1 cells in IMQ-treated mice $\left(35 \pm 11, \mathrm{P}<10^{-6}\right.$ and $\left.7 \pm 4\right)$ as compared to control (vaseline) $(75 \pm 12$ and $7 \pm 2)$ and normal group ( $81 \pm 5$ and $\left.6 \pm 2\right)$. (C) Distribution of $\mathrm{T}-\mathrm{CD} 4^{+} / \mathrm{T}-\mathrm{CD} 8^{+}$ratio in IMQ-treated mice $(1.37 \pm 0.0 .26)$ as compared to control (vaseline) (1.51 \pm 0.12$)$ and normal group $(1.52 \pm 0.23)$. The results are presented as percentage from $\mathrm{CD} 3 \varepsilon^{+}$lymphocytes (mean $\left.\pm \mathrm{SD}\right)$. 


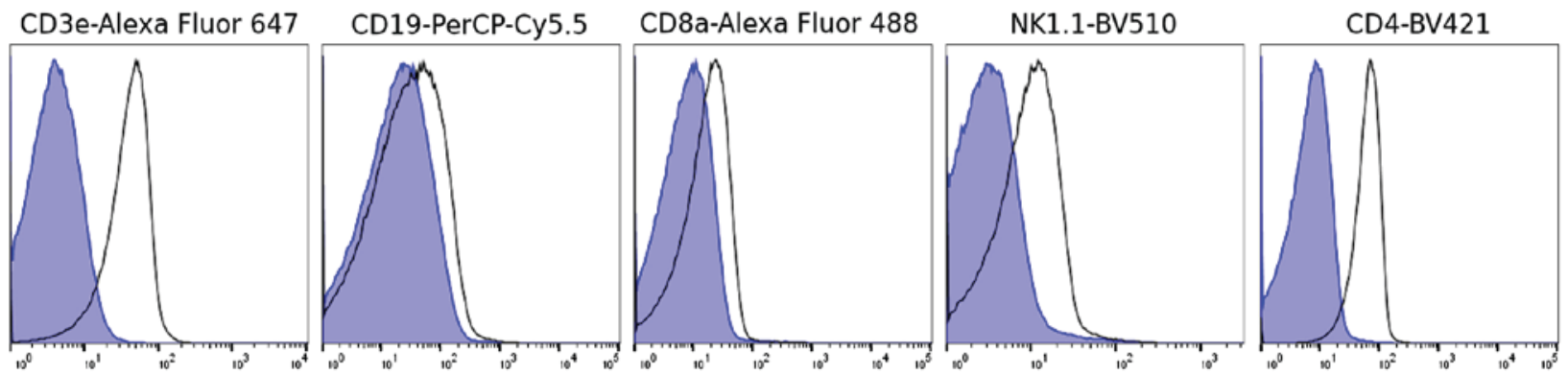

Figure 6. Analysis of fluorescence levels using Overton subtraction. An example of fluorescence data analysis; blue, unstained control; white, stained sample.

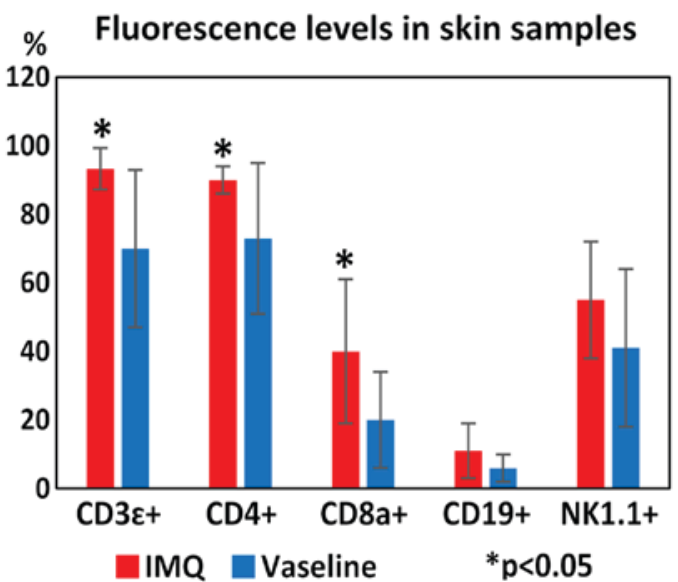

Figure 7. Immune cell pattern in skin samples. Expression of differences between mean Overton subtraction values in IMQ and vaseline-treated mice: CD $3 \varepsilon^{+}$cells $\left(93 \pm 6\right.$ vs. $\left.70 \pm 23,{ }^{*} \mathrm{P}<0.05\right)$; $\mathrm{CD} 4{ }^{+}$cells $\left(90 \pm 4\right.$ vs. $\left.73 \pm 22,{ }^{*} \mathrm{P}<0.05\right)$ CD8a ${ }^{+}$cells $\left(40 \pm 21\right.$ vs. $\left.20 \pm 14,{ }^{*} \mathrm{P}<0.05\right)$; $\mathrm{CD} 19^{+}$cells $(11 \pm 8$ vs. $6 \pm 4)$; NK1.1 cells $(55 \pm 17$ vs. $41 \pm 23)$. The results are presented as mean \pm SD.

mice vs. vaseline-treated mice) were statistically significant for $\mathrm{CD} 3 \varepsilon^{+}(93 \pm 6$ vs. $70 \pm 23$; $\mathrm{P}<0.05), \mathrm{CD}^{+}(90 \pm 4$ vs. $73 \pm 22$; $\mathrm{P}<0.05)$ and $\mathrm{CD}^{+} \mathrm{a}^{+}$cells $(40 \pm 21$ vs. $20 \pm 14 ; \mathrm{P}<0.05)$ (Fig. 7 ).

\section{Discussion}

Although there is an important imaging panel for investigating human disease (36-38), the need to find immunological pattern of this autoimmune disorders remains a constant issue. The studies related to the psoriatic animal model do not abound and comprise merely few dozen published papers. There have been attempts to establish a psoriatic-like mouse model, such as induction in immunodeficient (SCID) mice of psoriasiform lesions using staphylococcal enterotoxin B (SEB)-stimulated PBMCs (SEB-PBMCs) isolated from psoriatic patients as transplantation mouse psoriasis model. Another group transplanted in SCID mice skin harvested from psoriatic patients and afterwards both intradermal and intraperitoneal inoculations of bacterial superantigen was performed inducing a mouse model of psoriasis $(39,40)$. All these prior models were accompanied by technical difficulties including lack of reproducibility when human patient samples were transplanted. The first report on topical application of Aldara cream inducing an animal model of psoriatic lesion was published in 2009 (35) and continued in 2014 (41). In these reports the focused evaluations were performed mainly on the tissue pattern of inflammatory cells. In the monitoring domain of psoriasis evaluating both the circulatory and skin immune cells are equally important to further developing immune patterns that can monitor disease evolution and therapy efficacy.

We chose the murine model of psoriasiform dermatitis induced by topical IMQ application according to the prior described protocols (35) to further evaluate the immunological changes in lymphocyte populations from peripheral blood and spleen, and to estimate the amplitude of the cellular changes linked to the pathogenic mechanism. To our knowledge there are no similar studies regarding the immune cell characterization in blood, spleen and skin regarding IMQ experimental model. The on-site lesion echo of central changes was appreciated at the cell population level in lesioned tissue.

The immunological changes induced by IMQ treatment in lymphocyte populations from peripheral blood and spleen were evaluated by flow cytometry. The main changes observed in peripheral blood were the significantly increased of T-CD $8 \mathrm{a}^{+}$ lymphocytes and NK1.1+ cell percentages and the decreased of $\mathrm{T}-\mathrm{CD} 4^{+}$and $\mathrm{B}-\mathrm{CD} 19^{+}$lymphocyte percentages in IMQ-treated mice compared to control (vaseline) groups. As a consequence of the alteration of lymphocyte subpopulation distribution, the $\mathrm{T}-\mathrm{CD} 4^{+} / \mathrm{T}-\mathrm{CD} 8 \mathrm{a}^{+}$ratio is lower in mice treated with IMQ compared to control (vaseline) groups.

The increased percentage of T-CD8 lymphocytes in the peripheral circulation could suggest a cytotoxic mechanism at lesion level. At the origin of the auto-maintenance process in psoriasis are autoantigens and nucleic acids released by damaged epidermal cells (keratinocytes) that activate plasmacytoid dendritic cells. Recent studies show an abundance of proteins in the psoriatic skin mouse model that can account for the self-maintained auto-antigenic pool (42). The role of T-CD8 lymphocytes could be to recognize autoantigens and further destroy keratinocytes whose activation products are part of the auto-maintenance cycle of the disease. The interesting finding of peripheral NK cell increment associated with a decreased B lymphocyte percentage matches our prior reported results in other skin diseases (43) accounting for still unknown immune mechanisms of compensation between two types of immune cells.

In spleen samples, lymphocyte populations and subpopulations showed the same tendency of variation as in peripheral blood, but without statistical significance. The main change observed in spleen suspensions was the significant decrease of B cell percentages in the IMQ-treated mice compared to vaseline-treated mice and normal group. Decreased percentages of B lymphocytes in peripheral blood and spleen may suggest 
a general decrease in B lymphocytes as a result of a medullary dysfunction. It may also be possible for B lymphocytes to be arrested in certain lymphoid organs (e.g., lymph nodes) and/or digestive system, and subcutaneous tissues. As we found only a low expression of $\mathrm{CD} 19^{+}$cells in skin samples, it seems less likely B cells to be arrested at this level.

The values obtained for T-CD4 ${ }^{+}$lymphocyte percentages are low, both in peripheral blood and spleen suspensions. This may be due to the change in the proportion of Th subpopulations, but the results we have obtained so far do not allow us to pinpoint the cause of this decrease. The finding can be explained by an induced defective development of T-CD $4^{+}$ subpopulations with regulatory functions, immune cells that can contribute to the pathogenesis of psoriasis and/or other autoimmune diseases $(44,45)$.

Analysis of lymphocyte populations in skin samples revealed raised values of Overton subtraction for $\mathrm{CD} 3 \varepsilon^{+}$and $\mathrm{CD}^{+}{ }^{+}$cells (for both IMQ and vaseline-treated mice), and the differences between Overton subtraction values for experimental groups were statistically significant for $\mathrm{CD} 3 \varepsilon^{+}, \mathrm{CD} 4^{+}$ and $\mathrm{CD} \mathrm{a}^{+}$cells. These results may suggest the involvement of these cells in the lesional process. Moreover, as recently reported in the skin of mice exposed to IMQ a subset of IL-17-producing $\gamma \delta \mathrm{T}$ cells are endowed with long-lived memory $(45,46)$ thus we cannot exclude that there are several other immune subpopulations that contribute to the increased percentage of cells depicted by us in this model.

In conclusion, imiquimod-based murine model of psoriasiform dermatitis was analysed from an immunological point of view by performing a basic cellular profile at three levels: peripheral blood, spleen and skin. Expression of lymphocytes in these three immune areas may be a starting point for the specification of some immunological features of psoriasis with therapeutic utility. The evaluation was intended to establish the immune framework of this experimental model that could be further used for etio-pathogenic mechanisms identification and/or for studies regarding targeted therapies.

\section{Acknowledgements}

The present study will be integrated in the original part of $\mathrm{PhD}$ thesis of author PhD student Mihaela Surcel.

\section{Funding}

This study was supported by Grant PN-III-P1-1.2PCCDI-2017-0341/2018 and projects PN 18.21.02.01 and PN 18.21.02.02 and financed by Executive Agency for Higher Education, Research, Development and Innovation (Bucharest, Romania).

\section{Availability of data and materials}

The data sets used and/or analysed during the present study are available from the corresponding author on reasonable request.

\section{Authors' contributions}

RIH, MS, CC, MN and CU contributed to the creation and design of the research, acquisition, analysis and interpretation of the data, statistical analysis, drafting the manuscript, critical revision of the manuscript for important intellectual content. ANM, GI, IRP, DC, OB and CC were responsible for data acquisition, analysis and interpretation of data, and contributed to drafting and revising critically the manuscript for important intellectual content. All the authors read and approved the final version of the manuscript.

\section{Ethics approval and consent to participate}

The study protocol was approved by the Ethics Committee of 'Victor Babeș' National Institute of Pathology (Bucharest, Romania).

\section{Patient consent for publication}

Not applicable.

\section{Competing interests}

The authors declare that they have no competing interests.

\section{References}

1. Gudjonsson JE, Ding J, Li X, Nair RP, Tejasvi T, Qin ZS, Ghosh D, Aphale A, Gumucio DL, Voorhees JJ, et al: Global gene expression analysis reveals evidence for decreased lipid biosynthesis and increased innate immunity in uninvolved psoriatic skin. J Invest Dermatol 129: 2795-2804, 2009.

2. Parisi R, Symmons DP, Griffiths CE and Ashcroft DM; Identification and Management of Psoriasis and Associated ComorbidiTy (IMPACT) project team: Global epidemiology of psoriasis: A systematic review of incidence and prevalence. J Invest Dermatol 133: 377-385, 2013.

3. Horn EJ, Fox KM, Patel V, Chiou CF, Dann F and Lebwohl M: Association of patient-reported psoriasis severity with income and employment. J Am Acad Dermatol 57: 963-971, 2007.

4. Mease PJ and Armstrong AW: Managing patients with psoriatic disease: The diagnosis and pharmacologic treatment of psoriatic arthritis in patients with psoriasis. Drugs 74: 423-441, 2014.

5. Dommasch ED, Li T, Okereke OI, Li Y, Qureshi AA and Cho E: Risk of depression in women with psoriasis: A cohort study. Br J Dermatol 173: 975-980, 2015.

6. Ferreira BI, Abreu JL, Reis JP and Figueiredo AM: Psoriasis and associated psychiatric disorders: A systematic review on etiopathogenesis and clinical correlation. J Clin Aesthet Dermatol 9: 36-43, 2016.

7. Kölliker Frers RA, Bisoendial RJ, Montoya SF, Kerzkerg E, Castilla R, Tak PP, Milei J and Capani F: Psoriasis and cardiovascular risk: Immune-mediated crosstalk between metabolic, vascular and autoimmune inflammation. IJC Metab Endocr 6: 43-54, 2015.

8. Armstrong AW, Harskamp CT and Armstrong EJ: The association between psoriasis and obesity: A systematic review and metaanalysis of observational studies. Nutr Diabetes 2: e54, 2012.

9. Ganguly S, Ray L, Kuruvila S, Nanda SK and Ravichandran K: Lipid accumulation product index as visceral obesity indicator in psoriasis: A case-control study. Indian J Dermatol 63: 136-140, 2018.

10. Cohen AD, Dreiher J, Shapiro Y, Vidavsky L, Vardy DA, Davidovici B and Meyerovitch J: Psoriasis and diabetes: A population-based cross-sectional study. J Eur Acad Dermatol Venereol 22: 585-589, 2008.

11. Ahlehoff O, Gislason GH, Lindhardsen J, Charlot MG, Jørgensen CH, Olesen JB, Bretler DM, Skov L, Torp-Pedersen C and Hansen PR: Psoriasis carries an increased risk of venous thromboembolism: A Danish nationwide cohort study. PLoS One 6: e18125, 2011.

12. Ayala-Fontánez N, Soler DC and McCormick TS: Current knowledge on psoriasis and autoimmune diseases. Psoriasis (Auckl) 6: 7-32, 2016.

13. Sarac G, Koca TT and Baglan T: A brief summary of clinical types of psoriasis. North Clin Istanb 3: 79-82, 2016. 
14. Langley RG and Ellis CN: Evaluating psoriasis with psoriasis area and severity index, psoriasis global assessment, and lattice system physician's global assessment. J Am Acad Dermatol 51: 563-569, 2004.

15. Finlay AY and Khan GK: Dermatology Life Quality Index (DLQI) - a simple practical measure for routine clinical use. Clin Exp Dermatol 19: 210-216, 1994.

16. Mrowietz U and Reich K: Psoriasi - new insights into pathogenesis and treatment. Dtsch Arztebl Int 106: 11-19, 2009.

17. Caruntu C, Boda D, Dumitrascu G, Constantin C and Neagu M: Proteomics focusing on immune markers in psoriatic arthritis. Biomarkers Med 9: 513-528, 2015.

18. Lande R, Botti E, Jandus C, Dojcinovic D, Fanelli G, Conrad C, Chamilos G, Feldmeyer L, Marinari B, Chon S, et al: The antimicrobial peptide LL37 is a T-cell autoantigen in psoriasis. Nat Commun 5: 5621, 2014.

19. Krueger JG: An autoimmune 'attack' on melanocytes triggers psoriasis and cellular hyperplasia. J Exp Med 212: 2186-2186, 2015.

20. Benson JM, Sachs CW, Treacy G, Zhou H, Pendley CE, Brodmerkel CM, Shankar G and Mascelli MA: Therapeutic targeting of the IL-12/23 pathways: Generation and characterization of ustekinumab. Nat Biotechnol 29: 615-624, 2011.

21. Raţiu MP, Purcărea I, Popa F, Purcărea VL, Purcărea TV, Lupuleasa D and Boda D: Escaping the economic turn down through performing employees, creative leaders and growth driver capabilities in the Romanian pharmaceutical industry. Farmacia 59: 119-130, 2011.

22. Negrei C, Caruntu C, Ginghina O, Dragomiroiu GT, Toderescu CD and Boda D: Qualitative and quantitative determination of methotrexate polyglutamates in erythrocytes by high performance liquid chromatography. Rev Chim 66: 607-610, 2015.

23. Boda D, Negrei C, Nicolescu F and Balalau C: Assessment of some oxidative stress parameters in methotrexate treated psoriasis patients. Farmacia 62: 704-710, 2014.

24. Negrei C, Arsene AL, Toderescu CD, Boda D and Ilie M: Acitretin treatment in oisturiz may influence the cell membrane fluidity. Farmacia 60: 767-771, 2012.

25. Negrei C, Ginghină O, Căruntu C, Burcea Dragomiroiu GT, Jinescu $G$ and Boda D: Investigation relevance of methotrexate polyglutamates in biological systems by high performance liquid chromatography. Rev Chim 66: 766-768, 2015.

26. Olteanu R, Constantin MM,Zota A, Dorobantu DM, Constantin T, Serban ED, Balanescu P, Mihele D and Gheuca-Solovastru L: Original clinical experience and approach to treatment study with interleukine 12/23 inhibitor in moderate-to-severe psoriasis patients. Farmacia 64: 918-992, 2016.

27. Carrascosa JM, Jacobs I, Petersel D and Strohal R: Biosimilar drugs for psoriasis: Principles, present, and near future. Dermatol Ther (Heidelb) 8: 173-194, 2018.

28. Olteanu R, Zota A and Constantin M: Biosimilars: An update on clinical trials (review of published and ongoing studies). Acta Dermatovenerol Croat 25: 57-66, 2017.

29. Schön MP and Schön M: Imiquimod: Mode of action. Br J Dermatol 157 (Suppl 2): 8-13, 2007.

30. Hemmi H, Kaisho T, Takeuchi O, Sato S, Sanjo H, Hoshino K, Horiuchi T, Tomizawa H, Takeda K and Akira S: Small anti-viral compounds activate immune cells via the TLR7 MyD88-dependent signaling pathway. Nat Immunol 3: 196-200, 2002.

31. Urosevic M, Maier T, Benninghoff B, Slade H, Burg G and Dummer R: Mechanisms underlying imiquimod-induced regression of basal cell carcinoma in vivo. Arch Dermatol 139: 1325-1332, 2003.
32. Kono T, Kondo S, Pastore S, Shivji GM, Tomai MA, McKenzie RC and Sauder DN: Effects of a novel topical immunomodulator, imiquimod, on keratinocyte cytokine gene expression. Lymphokine Cytokine Res 13: 71-76, 1994.

33. Frotscher B, Anton K and Worm M: Inhibition of IgE production by the imidazoquinoline resiquimod in nonallergic and allergic donors. J Invest Dermatol 119: 1059-1064, 2002.

34. Council NR: Guide for the Care and Use of Laboratory Animals. 8th edition. National Academies Press, Washington, DC, pp1-133, 2010.

35. van der Fits L, Mourits S, Voerman JS, Kant M, Boon L, Laman JD, Cornelissen F, Mus AM, Florencia E, Prens EP, et al: Imiquimod-induced psoriasis-like skin inflammation in mice is mediated via the IL-23/IL-17 axis. J Immunol 182: 5836-5845, 2009.

36. Batani A, Brănisteanu DE, Ilie MA, Boda D, Ianosi S, Ianosi G and Caruntu C: Assessment of dermal papillary and microvascular parameters in psoriasis vulgaris using in vivo reflectance confocal microscopy. Exp Ther Med 15: 1241-1246, 2018.

37. Căruntu C, Boda D, Căruntu A, Rotaru M, Baderca F and Zurac S: In vivo imaging techniques for psoriatic lesions. Rom J Morphol Embryol 55 (Suppl 3): 1191-1196, 2014.

38. Căruntu C, Boda D, Musat S, Căruntu A and Mandache E: Stress-induced mast cell activation in glabrous and hairy skin. Mediators Inflamm 2014: 105950, 2014.

39. Yamamoto T, Matsuuchi M, Katayama I and Nishioka K: Repeated subcutaneous injection of staphylococcal enterotoxin B-stimulated lymphocytes retains epidermal thickness of psoriatic skin-graft onto severe combined immunodeficient mice. J Dermatol Sci 17: 8-14, 1998.

40. Boehncke WH, Zollner TM, Dressel D and Kaufmann R: Induction of psoriasiform inflammation by a bacterial superantigen in the SCID-hu xenogeneic transplantation model. J Cutan Pathol 24: 1-7, 1997.

41. Wohn CT, Pantelyushin S, Ober-Blöbaum JL and Clausen BE: Aldara-induced psoriasis-like skin inflammation: Isolation and characterization of cutaneous dendritic cells and innate lymphocytes. Methods Mol Biol 1193: 171-185, 2014

42. Lundberg KC, Fritz Y, Johnston A, Foster AM, Baliwag J, Gudjonsson JE, Schlatzer D, Gokulrangan G, McCormick TS, Chance MR, et al: Proteomics of skin proteins in psoriasis: From discovery and verification in a mouse model to confirmation in humans. Mol Cell Proteomics 14: 109-119, 2015.

43. Neagu M, Constantin C and Zurac S: Immune parameters in the prognosis and therapy monitoring of cutaneous melanoma patients: Experience, role, and limitations. Biomed Res Int 2013: 107940, 2013

44. Manda G, Neagu M, Livescu A, Constantin C, Codreanu C and Radulescu A: Imbalance of peripheral B lymphocytes and NK cells in rheumatoid arthritis. J Cell Mol Med 7: 79-88, 2003.

45. Singh K, Gatzka M, Peters T, Borkner L, Hainzl A, Wang H, Sindrilaru A and Scharffetter-Kochanek K: Reduced CD18 levels drive regulatory $\mathrm{T}$ cell conversion into Th17 cells in the CD18hypo PL/J mouse model of psoriasis. J Immunol 190: 2544-2553,2013.

46. Prinz I and Sandrock I: $\gamma \delta \mathrm{T}$ cells come to stay: Innate skin memory in the Aldara model. Eur J Immunol 45: 2994-2997, 2015.

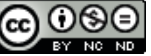

This work is licensed under a Creative Commons Attribution-NonCommercial-NoDerivatives 4.0 International (CC BY-NC-ND 4.0) License. 